\begin{tabular}{ccc}
\hline International Journal of Engineering \& Technology, $7(4.30)(2018) 281-284$ \\
SPC \\
Website w ww. sciencepubco.com/index.php/IJET \\
Research paper
\end{tabular}

\title{
Fuzzy Time Series Forecasting Model based on Frequency Density and Similarity Measure Approach
}

\author{
Nazirah Ramli ${ }^{1 *}$, Siti Musleha Ab Mutalib², Daud Mohamad ${ }^{3}$ \\ ${ }^{1}$ Faculty of Computer and Mathematical Sciences, Universiti Teknologi MARA Pahang, 26400, Bandar Jengka, Pahang, Malaysia \\ ${ }^{2,3}$ Faculty of Computer and Mathematical Sciences, Universiti Teknologi MARA Shah Alam, 40450, Shah Alam, Selangor, Malaysia \\ *Corresponding author E-mail: nazirahr@pahang.uitm.edu.my
}

\begin{abstract}
This paper proposes an enhanced fuzzy time series (FTS) prediction model that can keep some information under a various level of confidence throughout the forecasting procedure. The forecasting accuracy is developed based on the similarity between the fuzzified historical data and the fuzzy forecast values. No defuzzification process involves in the proposed method. The frequency density method is used to partition the interval, and the area and height type of similarity measure is utilized to get the forecasting accuracy. The proposed model is applied in a numerical example of the unemployment rate in Malaysia. The results show that on average $96.9 \%$ of the forecast values are similar to the historical data. The forecasting error based on the distance of the similarity measure is 0.031 . The forecasting accuracy can be obtained directly from the forecast values of trapezoidal fuzzy numbers form without experiencing the defuzzification procedure.
\end{abstract}

Keywords: Area and Height Similarity Measure; Forecasting Accuracy; Frequency Density; Fuzzy Time Series; Unemployment Rate.

\section{Introduction}

To overcome the drawback in the classical time series method, [1] proposed the fuzzy time series (FTS) prediction model. The discrete fuzzy set was used to represent the time series data, and the forecast value in terms of discrete fuzzy set was produced. A large number of studies have been carried out to improve the procedure of FTS in [1] such as by [2-4]. [2] proposed a model to improve the length of the interval by utilizing a new method and [3] proposed the FTS forecasting model which can deal with seasonal time series data. In another study, [4] proposed a higher orderforecasting model based on automatic grouping strategy and generalized FLR.

[5-6] used trapezoidal fuzzy numbers (TrFNs) to denote the linguistic term of the data, and produced the forecast values of TrFNs form. [1-6] defuzzified the forecast values to crisp values, and the forecasting accuracy such as mean absolute percentage error (MAPE), mean square error (MSE), and root mean square error (RMSE) was calculated. The defuzzification procedure produces the forecast values of single point form, and thus some information under a various level of confidence that kept throughout the forecasting procedure has been dissipated from the data. Figure 1 shows the process for obtaining the forecasting accuracy for [1-6].

This paper proposes an improved fuzzy forecasting model based on frequency density [7], and area and height similarity measure [8]. The frequency density partitioning method redefines the intervals based on the frequency of data onto each interval. This partitioning method reflects the distribution of data, and discards the interval of no distribution of data. The similarity measure concept, which portrays the level of likeness between two comparing objects, is generally utilized as a part of numerous applications, for example, pattern recognition, decision-making, and machine learning. In this study, the similarity measure is applied to compare the similarity between the forecast values and historical values. The forecasting accuracy of this FTS model is based totally on the degree of similarity between the forecast values and historical values.

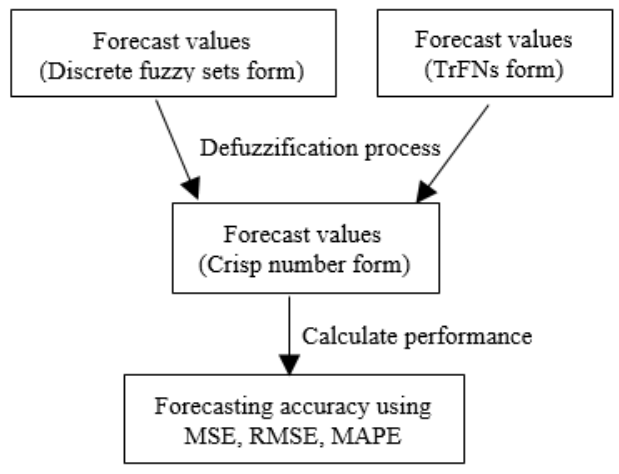

Fig. 1: The process for obtaining the forecasting accuracy from the previous methods [1-6]

This paper is organized as follows: The basic definition of FTS, TrFNs, and fuzzy similarity measure are presented in section two. Section three presents the proposed FTS model based on frequency density and similarity measure; section four illustrates the proposed technique by using the data of unemployment rate in Malaysia. The conclusion is presented in section five. 


\section{Preliminaries}

This section briefly reviewed some fundamental concepts on FTS $[1,5,9]$, TrFNs $[10]$ and the similarity measure of area and height type [8].

\section{Definition 1:}

Let $Y(t)(t=\ldots, 0,1,2, \ldots)$ be a subset of $\Re$ and $Y(t)$ be the universe of discourse defined by fuzzy set $\mu_{i}(t)(i=1,2, \ldots)$, then $H(t)$ is named as FTS on $Y(t)(t=\ldots, 0,1,2, \ldots),[9]$.

\section{Definition 2:}

Let $H(t)$ is an FTS. $H(t)$ is produced from $H(t-1)$ if there exists a fuzzy relationship $Q(t-1, t)$ such that $H(t)=H(t-1) \otimes Q(t-1, t)$ whereby $\otimes$ denotes as a fuzzy operator. The relationship can be denoted as $H(t-1) \rightarrow H(t)$, [9].

\section{Definition 3:}

Suppose that $H(t-1)=C_{i}$ and $H(t)=C_{j}$. The fuzzy logical relationship (FLR) can be defined as $C_{i} \rightarrow C_{j}$ where $C_{i}$ is the lefthand side and $C_{j}$ is the right-hand side of FLR. If the FLR on the left-hand side has the same fuzzy set, then the FLR can be further classified into the same FLR group, [5].

\section{Definition 4:}

A trapezoidal fuzzy number $(\operatorname{TrFN})$ denoted as $P=(p, q, r, s)$ is defined by the membership function as follows, [10]:

$$
\mu_{P}(x)=\left\{\begin{array}{cc}
0, & x<p \\
\frac{x-p}{q-p}, & p \leq x \leq q \\
1, & q \leq x \leq r \\
\frac{s-x}{s-r}, & r \leq x \leq s \\
0, & x>s
\end{array}\right.
$$

\section{Definition 5:}

Let $M=\left(m_{1}, m_{2}, m_{3}, m_{4} ; h_{M}\right)$ and $N=\left(n_{1}, n_{2}, n_{3}, n_{4} ; h_{N}\right)$ be two generalized TrFNs. The degree of similarity between $M$ and $N$ is denoted by $S(M, N)$ and defined as [8],

$$
\begin{aligned}
& S(M, N)=\left(1-\frac{1}{4} \sum_{i=1}^{4}\left|m_{i}-n_{i}\right|\right) \times\left(1-\frac{1}{2}\left\{|\operatorname{ar}(M)-\operatorname{ar}(N)|+\left|h_{M}-h_{N}\right|\right\}\right) \\
& \text { whereby } \operatorname{ar}(M) \text { is the area of TrFN } M \text { defines as } \\
& \operatorname{ar}(M)=\frac{\left(m_{4}+m_{3}-m_{2}-m_{1}\right) \times h_{M}}{2} .
\end{aligned}
$$

\section{Proposed Fuzzy Time Series Forecasting Model}

This section presents the proposed FTS forecasting model that consists of 10 steps. Steps 1 to 9 is the development of the model for producing the forecast values of TrFNs form. Step 10 is the process to calculate the forecasting accuracy (as shown in Figure 2).

Step 1: Collect the historical data $D_{t}$ and determine the minimum and maximum data denoted by $D_{\min }$ and $D_{\max }$ respectively.

Step 2: The universe of discourse is defined as $V=\left[D_{\min }-a_{1}, D_{\max }+a_{2}\right]$

whereby $a_{1}$ and $a_{2}$ are two appropriate positive real numbers.

Step 3: By using the randomly chosen length method, divide the universe of discourse $V$ into $m$ equal length intervals $v_{1}, v_{2}, v_{3}, \ldots$, $v_{m}$
Step 4: Count the frequency of the historical data included within the interval. Classify and sub-partition the interval based on their frequency density. The interval with the highest frequency is classified as Class 1 and is divided into four sub-partition. Table 1 shows the detailed classification and sub-partition of the interval.

\begin{tabular}{|c|c|c|} 
Table 1: Partition of Sub-interval \\
\begin{tabular}{|c|c|c|}
\hline $\begin{array}{c}\text { Level of Frequency Numbers of } \\
\text { Interval }\end{array}$ & Class & $\begin{array}{c}\text { Number of Sub- } \\
\text { interval }\end{array}$ \\
\hline Highest & 1 & 4 \\
\hline Second highest & 2 & 3 \\
\hline Third highest & 3 & 2 \\
\hline Fourth highest and above & 4 & 1 \\
\hline
\end{tabular}
\end{tabular}

Step 5: Based on Table 1, list all the new sub-intervals $w_{1}, w_{2}$, $w_{3}, \ldots, w_{k}$.

Step 6: Based on the new sub-intervals obtained in Step 5, establish the new TrFNs as follows:

$C_{1}=\left(d_{0}, d_{1}, d_{2}, d_{3}\right), C_{2}=\left(d_{1}, d_{2}, d_{3}, d_{4}\right), \ldots, C_{k-1}=\left(d_{k-2}, d_{k-1}, d_{k}, d_{k+1}\right)$, $C_{k}=\left(d_{k-1}, d_{k}, d_{k+1}, d_{k+2}\right)$.

Step 7: Transform the historical data $D_{t}$ to TrFNs form. If the value of the historical data is located in the range of $w k$, then it belongs to $\mathrm{TrFN} C_{k}$

Step 8: Establish the FLR and FLR group based on Definition 3.

Step 9: Based on the heuristic rules from [11], calculate the fuzzy forecasted value $H_{t}$ in the form of TrFNs. Normalize the $H_{t}$ and $D_{t}$. Step 10: Calculate the similarity of $H_{t}$ and $D_{t}$ by using area and height similarity measure approach from Definition 5 [8]. Then, calculate the forecasting error which is defined as

$D=1-\operatorname{avg} S(M, N)$ whereby $\operatorname{avg} S(M, N)$ is the average degree of similarity between $\operatorname{TrFNs} M$ and $N$.

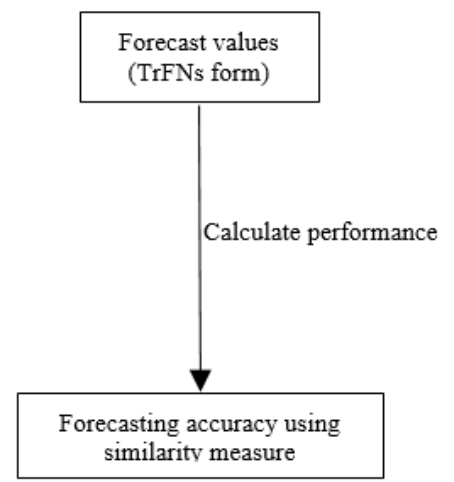

Fig. 2: The process for obtaining the forecasting accuracy for the proposed method

\section{Numerical Example}

The proposed FTS forecasting model is illustrated using the data of the unemployment rate in Malaysia from the year 1982 to 2013 [12] (shown in Figure 3).

Step 1: Based on the unemployment rate data from [12], $D_{\min }=$ $2.4 \%$ and $D_{\max }=7.4 \%$.

Step 2: By choosing two appropriate numbers as $a_{1}=0.4$ and $a_{2}=$ 0.6 , the universe of discourse is defined as $V=[2.0,8.0]$.

Step 3: By choosing at random the interval length as 0.75 , the universe of discourse $V$ is divided into eight equal length as follows: $v_{1}=[2,2.75], \quad v_{2}=[2.75,3.5], \quad v_{3}=[3.5,4.25], \quad v_{4}=[4.25,5]$, $v_{5}=[5,5.75], v_{6}=[5.75,6.5], v_{7}=[6.5,7.25], v_{8}=[7.25,8]$. 


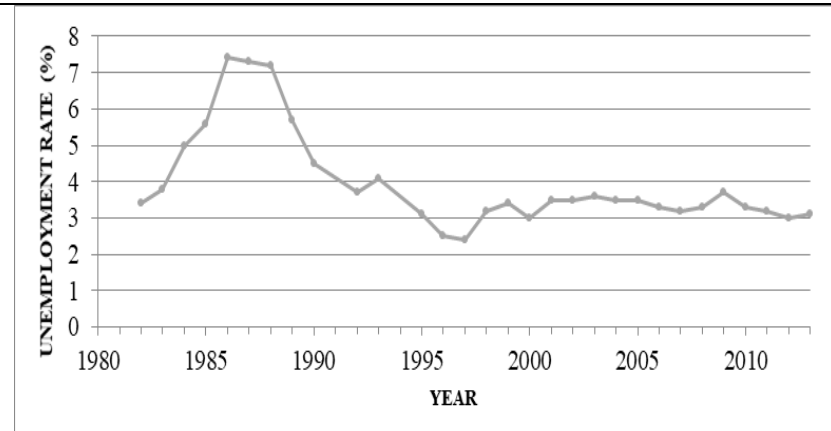

Fig. 3: Data of unemployment rate in Malaysia from 1982 to 2013 [12]

Step 4: The frequency, classification, and sub-interval are shown in Table 2.

Table 2: Classification and sub-interval of unemployment rate data

\begin{tabular}{|l|c|c|c|}
\hline \multicolumn{1}{|c|}{ Interval } & Frequency & Class & $\begin{array}{c}\text { Number of } \\
\text { Sub-interval }\end{array}$ \\
\hline$v_{1}=[2,2.75]$ & 2 & 3 & 2 \\
\hline$v_{2}=[2.75,3.5]$ & 16 & 1 & 4 \\
\hline$v_{3}=[3.5,4.25]$ & 7 & 2 & 3 \\
\hline$v_{4}=[4.25,5]$ & 2 & 3 & 2 \\
\hline$v_{5}=[5,5.75]$ & 2 & 3 & 2 \\
\hline$v_{6}=[5.75,6.5]$ & 0 & 4 & 1 \\
\hline$v_{7}=[6.5,7.25]$ & 1 & 4 & 1 \\
\hline$v_{8}=[7.25,8]$ & 2 & 3 & 2 \\
\hline
\end{tabular}

Step 5: Based on the number of sub-interval in Table 2, there are 17 new sub-intervals obtained which are $w_{1}=[2,2.375]$, $w_{2}=[2.375,2.75], \quad w_{3}=[2.75, \quad 2.938], \quad w_{4}=[2.938,3.125]$, $w_{5}=[3.125,3.313], \quad w_{6}=[3.313,3.5], \quad w_{7}=[3.5,3.75], \quad w_{8}=[3.75,4]$, $w_{9}=[4,4.25], \quad w_{10}=[4.25,4.625], \quad w_{11}=[4.625,5], \quad w_{12}=[5,5.375]$, $w_{13}=[5.375,5.75], w_{14}=[5.75,6.5], w_{15}=[6.5,7.25], w_{16}=[7.25,7.625]$, $w_{17}=[7.625,8]$.

Step 6: The linguistic term of unemployment rate in TrFNs form are given as follows:

$C_{1}=(1.625,2,2.375,2.75), C_{2}=(2,2.375,2.75,2.938), \ldots$, $C_{16}=(6.5,7.25,7.625,8), C_{17}=(7.25,7.625,8,8.375)$.

Step 7: Table 3 shows the fuzzified unemployment rate in Malaysia for the year 2008 to 2013.

Step 8: Table 4 presents the FLR group of the unemployment rate.

Table 3: Fuzzified unemployment rate in TrFNs form

\begin{tabular}{|c|c|c|c|}
\hline Year & $\begin{array}{c}\text { Unemployment } \\
\text { rate }\end{array}$ & TrFNs \\
\hline $\mathbf{2 0 0 8}$ & & 3.3 & $A_{5}$ \\
\hline $\mathbf{2 0 0 9}$ & & 3.7 & $A_{7}$ \\
\hline $\mathbf{2 0 1 0}$ & & 3.3 & $A_{5}$ \\
\hline $\mathbf{2 0 1 1}$ & & 3.2 & $A_{5}$ \\
\hline $\mathbf{2 0 1 2}$ & & 3 & $A_{4}$ \\
\hline $\mathbf{2 0 1 3}$ & & 3.1 & $A_{4}$ \\
\hline
\end{tabular}

Table 4: FLR group of unemployment rate

\begin{tabular}{|l|l|l|l|}
\hline Group & FLR & Group & FLR \\
\hline \multirow{4}{*}{2} & $C_{2} \rightarrow C_{2}, C_{2} \rightarrow C_{5}$ & 6 & $C_{8} \rightarrow C_{11}$ \\
& $C_{4} \rightarrow C_{2}, C_{4} \rightarrow C_{2}$, & 7 & $C_{9} \rightarrow C_{7}$ \\
3 & $C_{4} \rightarrow C_{2}$ & 8 & $C_{10} \rightarrow C_{7}, C_{10} \rightarrow C_{9}$ \\
& $C_{5} \rightarrow C_{4}, C_{5} \rightarrow C_{5}$, & 9 & $C_{11} \rightarrow C_{13}$ \\
4 & $C_{5} \rightarrow C_{6}, C_{5} \rightarrow C_{7}$ & 10 & $C_{13} \rightarrow C_{10}, C_{13} \rightarrow C_{16}$ \\
& $C_{6} \rightarrow C_{4}, C_{6} \rightarrow C_{5}$, & 11 & $C_{15} \rightarrow C_{13}$ \\
& $C_{6} \rightarrow C_{6}, C_{6} \rightarrow C_{5}$, & 12 & $C_{16} \rightarrow C_{15}, C_{16} \rightarrow C_{16}$ \\
5 & $C_{6} \rightarrow C_{8}$ & 13 & $C_{4} \rightarrow \phi$ \\
& $C_{7} \rightarrow C_{4}, C_{7} \rightarrow C_{5}$, & & \\
& $C_{7} \rightarrow C_{6}, C_{7} \rightarrow C_{9}$ & & \\
\hline
\end{tabular}

Step 9: Based on the heuristic rules from [11], the fuzzy forecast value $H_{t}$ is calculated. The values of $H_{t}$ for the year 2008 to 2013 are shown in Table 5. To normalize the historical data $C_{t}$ and forecast $H_{t}, C_{t}$ and $H_{t}$ are divided by 10 .
Table 5: Fuzzy forecasted unemployment rate for year 2008 until 2013

\begin{tabular}{|l|l|l|}
\hline Year & Fuzzy historical data & Fuzzy forecasted \\
\hline 2008 & $(2.938,3.125,3.313,3.5)$ & $(3.031,3.219,3.422,3.641)$ \\
2009 & $(3.313,3.5,3.75,4)$ & $(3.031,3.219,3.422,3.641)$ \\
2010 & $(2.938,3.125,3.313,3.5)$ & $(3.141,3.344,3.547,3.797)$ \\
2011 & $(2.938,3.125,3.313,3.5)$ & $(3.031,3.219,3.422,3.641)$ \\
2012 & $(2.75,2.938,3.125,3.313)$ & $(3.031,3.219,3.422,3.641)$ \\
2013 & $(2.75,2.938,3.125,3.313)$ & $(2.625,2.875,3.125,3.333)$ \\
\hline
\end{tabular}

Step 10: Based on the normalized $C_{t}$ and normalized $H_{t}$, the area and height similarity measure [8] is calculated as shown in Table 6.

Table 6: The area and height similarity measure (Year 2008- 2013)

\begin{tabular}{|c|c|c|c|c|c|}
\hline Year & Similarity & Year & Similarity & Year & Similarity \\
\hline 1983 & 0.953 & 1994 & 1 & 2004 & 0.995 \\
\hline 1984 & 1 & 1995 & 0.955 & 2005 & 0.997 \\
\hline 1985 & 1 & 1996 & 0.944 & 2006 & 0.976 \\
\hline 1986 & 0.851 & 1997 & 0.958 & 2007 & 0.988 \\
\hline 1987 & 0.963 & 1998 & 0.958 & 2008 & 0.988 \\
\hline 1988 & 0.963 & 1999 & 0.991 & 2009 & 0.966 \\
\hline 1989 & 1 & 2000 & 0.957 & 2010 & 0.974 \\
\hline 1990 & 0.851 & 2001 & 0.953 & 2011 & 0.988 \\
\hline 1991 & 1 & 2002 & 0.997 & 2012 & 0.969 \\
\hline 1992 & 1 & 2003 & 0.978 & 2013 & 0.990 \\
\hline 1993 & 0.924 & & & & \\
\hline
\end{tabular}

Table 6 shows the degree of similarity for the years 1984, 1985, 1989, 1991, 1992 and 1994 are equal to one. It demonstrates that $19.4 \%$ of the forecast unemployment rate is precisely similar with the actual value. $100 \%$ of the forecast values have more than $85 \%$ degree of similarity and on average the forecast values have $96.9 \%$ similarity with the actual value. The forecasting error based on the distance of the similarity measure is 0.031 compared to $7.62 \%$ for the MAPE value. According to [13], MAPE is the most helpful measurement to investigate the accuracy of forecasts between various elements as it measures relative performance. Based on the scale of forecasting accuracy from [13], the MAPE value indicates that the forecasting model has a good forecast. However, in order to obtain the MAPE values, the forecast values of TrFNs need to be transformed to crisp numbers via defuzzification process.

\section{Conclusion}

In this paper, the forecasting accuracy is developed based on the area and height type of similarity measure concept. The forecasting accuracy can be obtained directly from the forecast values of TrFNs form without experiencing the defuzzification procedure as compared to most of the previous studies of FTS such as [1-6] (as shown in Figures 1 and 2). The proposed FTS model preserves the forecast values of TrFNs form and thus able to keep some information under various level of confidence from being lost.

\section{Acknowledgement}

This research is supported by Ministry of Education Malaysia. (MOE) and Universiti Teknologi MARA under the Academic \& Research Assimilation 0092/2016.

\section{References}

[1] Song Q \& Chissom BS (1993), Forecasting enrollments with fuzzy time series - Part I. Fuzzy Sets and Systems 54, 1-9.

[2] Yu HK (2005), A refined fuzzy time series model for forecasting. Physica A: Statistical Mechanics and its Application 346(3-4), $657-$ 681.

[3] Liu HT \& Wei ML (2010), An improved fuzzy forecasting method for seasonal time series. Expert Systems with Applications 39(9), 6310-6318. 
[4] Qiu W, Zhang P \& Wang Y (2015), Fuzzy time series forecasting model based on automatic clustering techniques and generalized fuzzy logical relationship. Mathematical Problems in Engineering, $1-8$.

[5] Liu HT (2007), An improved fuzzy time series forecasting method using trapezoidal fuzzy numbers. Fuzzy Optimization and Decision Making 6(1), 63-80.

[6] Liu HT (2009), An integrated fuzzy time series forecasting system. Expert Systems with Applications 36 (6), 10045-10053.

[7] Hsu CC \& Chen SM (2002), A new method for forecasting enrollments based on fuzzy time series. Proceedings of the Seventh Conference on Artificial Intelligence and Applications, 17-22.

[8] Patra K \& Modal SK (2015), Fuzzy risk analysis using area and height based similarity measure on generalized trapezoidal fuzzy numbers and its application. Applied Soft Computing 28, 276-284.

[9] Song Q \& Chissom BS (1994), Forecasting enrollments with fuzzy time series - Part II. Fuzzy Sets and Systems 62, 1-8.

[10] Wang X (1997), An investigation into relations between some transitivity related concept. Fuzzy sets and Systems 89(2), 257-262.

[11] Cheng C, Wang J \& Li C (2008), Forecasting the number of outpatient visits using a new fuzzy time series based on weightedtransitional matrix. Expert Systems with Applications 34(4), 2568 2575.

[12] Department of Statistic Malaysia. Time series data of unemployment. https://www.dosm.gov.my. Accessed January 13, 2014

[13] Lewis CD, Industrial and business forecasting methods, Butterworths, London, (1982) 